\title{
PERCEPCIONES Y ACTITUDES SOBRE HOMOFOBIA EN ESTUDIANTES UNIVERSITARIOS
}

\section{PERCEPTIONS AND ATTITUDES ABOUT HOMOPHOBIA IN UNIVERSITY STUDENTS}

\author{
Linda Teresa Orcasita ${ }^{1}$, José Ángel Vera Noriega², Magdalena Kusserow ${ }^{3}$ y José Luis Montenegro Céspedes ${ }^{1}$ \\ ${ }^{1}$ Facultad de Humanidades y Ciencias Sociales. Pontificia Universidad Javeriana Cali. Colombia \\ ${ }^{2}$ Centro de Investigación en Alimentación y Desarrollo. México
}

${ }^{3}$ Universidad de Konstanz. Alemania

\section{Abstract}

Objective. This article aims to explore perceptions and attitudes about homophobia in university students. Method. From a quantitative perspective, 228 young people from a private university in Cali-Colombia participated, the information was collected through a questionnaire. The statistical analysis of the results was carried out by the t-student and ANOVA tests. Results. The results show significant differences between the variables gender, faculty, income and self-concept in homophobia scores. Male, engineering, high family income, and negative self-concept participants showed a higher level of homophobia than the rest of the participants. Conclusions. It is concluded that the participants relate homosexuality with low family income, and that the positive self-concept favors the perception and attitude towards homosexual people.

Keywords: Attitudes, homophobia, homosexuality, youth, social perception.

\section{Resumen}

Objetivo. Este artículo tiene como objetivo explorar las percepciones y actitudes sobre homofobia en estudiantes universitarios. Método. Desde una perspectiva cuantitativa, se contó con la participación de 228 jóvenes de una universidad privada de CaliColombia, la recolección de información se hizo a través de un cuestionario. El análisis estadístico de los resultados se realizó por las pruebas t-studenty ANOVA. Resultados. Los resultados muestran diferencias significativas entre las variables género, facultad, ingresos y autoconcepto en las puntuaciones de homofobia. Los participantes masculinos, de ingeniería, con ingresos familiares altos y con un autoconcepto negativo, mostraron un nivel de homofobia más alto que el resto de los participantes. Conclusiones. Se concluye que los participantes relacionan la homosexualidad con ingresos familiares bajos, y que el autoconcepto positivo favorece la percepción y actitud hacia las personas homosexuales.

Palabras clave: Actitudes, homofobia, homosexualidad, jóvenes, percepción social.

Correspondencia: Linda Teresa Orcasita

Grupo de Investigación Bienestar, Trabajo, Cultura y Sociedad, Departamento de Ciencias Sociales.

Itorcasita@javerianacali.edu.co 
La expresión de las diferentes orientaciones e identidades sexuales ha generado que se construyan políticas en donde prevalezca el ejercicio de igualdad y equidad en derechos, de las personas que se reconocen como gais, lesbianas y bisexuales (LGB). Sin embargo, diversas investigaciones indican que adultos y jóvenes LGB tienen un alto riesgo de sufrir situaciones asociadas con la salud mental, en comparación con las personas heterosexuales (Shilo \& Mor, 2014), lo cual puede estar influido por las experiencias de discriminación, rechazo y exclusión frente a la diversidad sexual y de géneros (Hatzenbuehler, McLaughlin, Keyes \& Hasin, 2010).

Estas situaciones y experiencias de discriminación pueden ser producto de la presencia de homofobia que coexiste en la sociedad. El término "homofobia" se ha utilizado ampliamente para la conceptualización de la violencia y la discriminación contra las personas LGB (Junqueira, 2012; Prado, Aurelio, Mountian, Machado \& Cardoso dos Santos, 2010). La pregunta es ¿qué refleja este término? ¿Una patología, una actitud, una personalidad o una cuestión política, cultural, histórica, psicológica y sociológica? El término científico de homofobia es definido como una actitud negativa e irracional contra las personas homosexuales, incluyendo una aversión e interacción con personas homosexuales (Silva, Jaeger \& Valdivia-Moral, 2018), la cual puede estar expresada o sentida en personas heterosexuales y homosexuales (endodiscriminación).

Adicionalmente, autores como Rodríguez-Otero \& Treviño-Martínez (2016) han afirmado que la homofobia procede de las actitudes sexistas, pues estas refieren a la actitud hostil y/o benevolente hacia los géneros en virtud de la pertenencia grupal a un sexo biológico, siendo aquel donde existe una subordinación cognitiva, afectiva y conductual respecto alguno de los sexos. Estas actitudes son uno de los fundamentos por el cual se generan actitudes homofóbicas, tal como se evidencia en su estudio (Rodríguez-Otero \& Treviño-Martínez, 2016) en el cual encontraron que las actitudes sexistas están directamente relacionadas con los niveles de rechazo hacia las personas LGTBI y a su vez entre los distintos tipos de rechazo según la orientación o identidad sexual.

La homofobia generalmente se genera a partir de los mitos, creencias y estereotipos (Aggleton \& Parker, 2002) con los que operan las personas, y esta puede ser expresada de dos formas, implícita o explícitamente, de forma diferente en los individuos. La primera, se refiere a las actitudes y atracciones cognitivas que se establecen y desarrollan en la niñez. La homofobia implícita ha sido objeto de estudio en varias investigaciones, en las cuales se ha utilizado el instrumento del Implicit Association Test (IAT) (Boysen, Vogel \& Madon, 2006; Rohner \& Björklund, 2006; Tsang \& Rowatt, 2007). Mientras la homofobia explícita es expresada en actitudes conscientes, expresiones y actos contra personas que se reconocen con alguna orientación homosexual o bisexual. Para identificar este tipo de homofobia en investigación se recurre a cuestionarios de autoreporte.

Como se ha mencionado anteriormente, la homofobia puede generar actos de discriminación (exclusión social, rechazo en instituciones educativas y empleos; vulneración en los derechos como el matrimonio o la adopción, exigibilidad para someterse a exámenes de VIH, entre otros) (Aggleton \& Parker, 2002) y violencia física, psicológica y social a personas LGB, y en casos extremos se pude llegar incluso al homicidio. En Latinoamérica es poco el seguimiento registrado que se tiene, debido a razones como el miedo a denunciar dichos actos violentos o discriminatorios a personas LGB a las autoridades. Sin embargo, en Brasil, en el año 2012, se reportaron 310 homicidios de personas LGB asociados a la discriminación (Mastropasqua, 2015). Así pues, a raíz de estos actos de discriminación y violencias las personas LGB están expuestas a mayor vulneración de sus derechos, lo cual puede generar afectaciones en su calidad de vida a nivel psicosocial, físico, económico y político (Rodríguez-Otero \& Treviño-Martínez, 2016).

De acuerdo con la concepción teórica psicodinámica, la homofobia aumenta en las personas que tienen una atracción hacia el mismo sexo, que se podría denominar homofobia internalizada, lo cual es una fuente principal para el desarrollo de la misma que provoca ansiedad por la atracción inconsciente (Navarro \& Mebarak, 2014). No obstante, diversas investigaciones no han podido corroborar la concepción psicodinámica a través del instrumento IAT para medir las atracciones implícitas de los participantes (Lazarevic, Orlić \& Knežević, 2015; Mahaffey, Bryan, Ito \& Hutchison, 2011), pues no encontraron alguna asociación entre atracción implícita hacia el mismo sexo y la homofobia autoreportada.

Por otro lado, las personas homosexuales tienen un alto riesgo de ser acosadas de manera física, verbal, social 
o de manera virtual por su orientación sexual e incluye un abuso sistemático de poder que se caracteriza por su intencionalidad, frecuencia y diferencias de poder de los actores (Varjas, Dew, Marshal, Graybill, Singh, Meyers \& Birckbichler, 2008; O'Malley, Kann, Vivolo-Kantor, Kinchen \& McManus, 2014; Rodríguez-Otero, 2018). El acoso basado en la orientación sexual implícito o explicito se relaciona con algunos problemas de salud en adolescentes y adultos incluyendo; a) síntomas depresivos; b) una menor calidad de vida; c) ansiedad; d) alteraciones de comportamiento y e) problemas de autoestima (Andreou, Didaskalou \& Vlachou, 2008; Umana-Taylor \& Updegraff, 2007; Evans, Smokowski \& Cotter, 2014).

Algunos estudios identifican cómo el contexto sociocultural de la heterosexualidad obligatoria es central en la comprensión de los relatos del estrés de las minorías y la construcción de la identidad. Las jóvenes muestran más fluidez con respecto a sus sentimientos, comportamientos e identidades sexuales en contextos no heteronormativos. La invisibilidad de las mujeres lesbianas como identidad o práctica conduce a la confusión sobre lo que significaban los sentimientos para el futuro en el ámbito de la experiencia vivida (Dempsey, Hillier, \& Harrison, 2002; Flowers \& Buston, 2001). Por otro lado, las actitudes implícitas de participantes femeninas hacia las lesbianas eran generalmente positivas al igual que sus actitudes hacia los heterosexuales (Steffens, 2005). Igualmente es importante destacar las micro agresiones; comentarios breves y muy comunes que reflejan algún tipo de injuria, rechazo, diferenciación, sean intencionales o no, que comunican hostilidad, desprecio o insultos hacia un grupo minoritario, incluyendo personas de diferentes grupos étnicos, mujeres, personas LGB, minorías religiosas o personas en situación de discapacidad, lo cual afecta de manera negativa la salud mental (Nadal \& Griffin, 2011).

Por otra parte, las creencias, valores y roles tradicionales de género influyen en la homofobia que construyen las personas heterosexuales como no heterosexuales. Por ejemplo, la "hipermasculinidad" es expresada y reafirmada en los hombres, y cuando algún hombre sale de los parámetros establecidos los demás pueden expresar y sentir homofobia. De acuerdo con lo que plantean Moral \& Valle (2014), los hombres heterosexuales muestran más actitudes hostiles contra personas LGB, especialmente a hombres gais, esto se puede deber a lo que concluyen Nagoshi, Adams, Terrell, Hill \& Brzuzy (2008), quienes afirman que la homofobia esta correlacionada con un autoritarismo radical, el fundamentalismo religioso y el sexismo. Un estudio cuantitativo en Paraguay mostró que la distancia social (pertenecer a grupos sociales, relaciones, vivir con pareja, etc.) hacia gais y lesbianas tiene una correlación positiva con el prejuicio contra la población LGB (Coppari et al., 2014). Eso indica que la integración de personas LGB en grupos sociales o comunidades puede conducir a menos prejuicios contra ellos mismos.

Las personas LGB también pueden tener o sentir homofobia, la cual se denomina particularmente como homofobia internalizada (HI). Este tipo de homofobia puede ser causada por los parámetros establecidos socialmente frente a las relaciones afectivas y sexuales entre hombres y mujeres. Meyer \& Dean (1998) definen la $\mathrm{HI}$ como la representación interna de las actitudes homofóbicas de la sociedad en los individuos LGB, las cuales se componen hacia su propia orientación sexual, actitudes negativas hacia la homosexualidad, incomodidad con la revelación y expresión de la orientación sexual y de la actividad sexual, así como discriminar a otras personas LGB.

La HI en personas LGB produce afecciones negativas en su salud mental, como baja autoestima, depresión, ansiedad o ideación suicida (António \& Moleiro, 2015). Por su parte, a mayor nivel de HI, se obstaculiza la construcción de relaciones interpersonales dado que se puede presentar violencia de pareja (Badenes-Ribera, Sánchez-Meca \& Longobardi, 2017), consumo de sustancias psicoactivas (Kelly, Davis \& Schilesinger, 2015), prácticas sexuales sin protección que pueden tener como consecuencia la transmisión de ITS/VIH (Jeffries, Marks, Lauby, Murrill \& Millett, 2013).

Sin embargo, poder disminuir o resolver la $\mathrm{HI}$ con el tiempo causa resultados positivos para la salud de las personas LGB (Herrick et al., 2013). Teorías sobre el desarrollo de la identidad en personas LGB indican que la $\mathrm{HI}$ es frecuentemente experimentada en el proceso del desarrollo de la identidad y que superar la $\mathrm{HI}$ es esencial para el desarrollo de un autoconcepto saludable (Fingerhut, Peplau \& Ghavami, 2005).

A partir de lo anterior, se deduce que es importante diseñar investigaciones e intervenciones que exploren e 
intervengan sobre la aceptación, revelación y expresión individual y social de la orientación sexual diversa, pues contribuye a promover la salud mental y sexual de personas LGB, como lo menciona Formby (2011), quien brinda algunas recomendaciones para prácticas saludables en las relaciones sexuales para jóvenes de todas las identidades y orientaciones sexuales.

Ahora bien, poco se sabe sobre las actitudes de jóvenes universitarios respecto a personas con diversas orientaciones sexuales, es por ello que la presente investigación tuvo como objetivo explorar las percepciones y actitudes sobre la homosexualidad en estudiantes universitarios, y cómo se relacionan con sus características sociodemográficas.

\section{MÉTODO}

\section{Diseño y Participantes}

La presente investigación tuvo una perspectiva cuantitativa con un diseño no experimental ex post facto de corte transversal, el alcance del análisis estadístico es de tipo descriptivo y analítico (HernándezSampieri, Fernández \& Baptista, 2010). El tipo de muestreo utilizado para el trabajo es no probabilístico donde el alumno es la unidad de análisis y las comparaciones proceden de los atributos personales y escolares. El muestreo de máxima variabilidad trata de que la muestra incluya las diferentes posibilidades de las variables más importantes. En este caso, se realizó una evaluación de las actitudes a la homofobia en una universidad y se estimó encontrar diferencias significativas entre alumnos y alumnas en carreras tradicionalmente masculinas o feminizadas. La comparación entre los casos se llevó a cabo en diferentes áreas y con un número semejante de repeticiones, con el objeto de identificar patrones comunes de importancia que cortan transversalmente las variaciones. Se contó con la participación de 228 jóvenes universitarios/as (84 hombres, 140 mujeres 4 datos perdidos) pertenecientes a las Facultades de Humanidades y Ciencias Sociales, Ciencias Económicas y Administrativas, Ingeniera y Ciencias de la Salud de una universidad privada de la ciudad de Cali-Colombia. La edad de los y las estudiantes osciló entre 18 y 20 años. Por su parte, los ingresos familiares de los participantes fueron distribuidos equilibradamente entre "menos de 2
SMMLV" y "más de 10 SMMLV" (SMMLV = Salario Mínimo Mensual Legal Vigente) y se encontraban, en su mayoría, entre tercer o cuarto semestre de pregrado.

\section{Instrumentos}

El instrumento utilizado en la presente investigación contiene, en primer lugar, información sociodemográfica como: género, edad, ingresos familiares, semestre y facultad; y cinco escalas de medición.

Escala del Rechazo a la intimidad. Parte de la escala de Pettigrew \& Meertens (1995), la cual examina los aspectos más superlativos del etno-racismo que permitió ser un punto de partida para el diseño de una medida para estudiar el prejuicio contra la homosexualidad. A partir de lo anterior, la escala estuvo conformada por cinco ítems que describen situaciones cotidianas relacionadas al rechazo de relaciones íntimas de personas homosexuales. Los/as estudiantes indicaron el nivel de incomodidad con cada una de esas situaciones en una escala de 1 no me incomoda hasta 5 me incomoda mucho. Con base en un análisis factorial, se evidenció que los cinco ítems cargan en un factor con 57\% de varianza explicada y la precisión de la medida fue confirmada con un valor de Alpha de Cronbach de .86.

Escala de la Expresión Emocional. Se basó en la escala de Dijker (1987) para examinar las dimensiones emocionales incluidas en el prejuicio. Los participantes tenían una lista con tres emociones positivas (admiración, respeto y amor) y tres emociones negativas (desprecio, rabia y enojo), y debían escoger la intensidad de las emociones sentidas de acuerdo a las relaciones homosexuales, indicando en una escala de 1 Nunca a 5 Muchas veces. Los resultados del análisis factorial mostraron que los ítems con las emociones positivas explican en un $22 \%$ de la variabilidad y los ítems con las emociones negativas explican $27 \%$ de la varianza total. La consistencia interna de los ítems de las emociones positivas es aceptable con un valor de Alpha de Cronbach de .79, y los ítems de las emociones negativas tienen un valor de .67. Lacerda, Pereira \& Camino (2002) reportan que estos valores de consistencia interna son suficientes para indicar expresiones emocionales al respecto de la homosexualidad.

Escala de explicaciones de la homosexualidadEvaluación de un conjunto de ítems representando cinco causas posibles de la homosexualidad, los cuales 
permitieron medir la adhesión de los participantes a las diferentes explicaciones de la homosexualidad evaluado con una escala de 1 Nunca a 5 Siempre. Las cinco explicaciones fueron: (a) Explicaciones biológicas: Por ejemplo "las causas de la homosexualidad están relacionadas con disfunciones hormonales"; (b) Explicaciones éticas, como "las causas de la homosexualidad están relacionadas con la falta de respeto"; (c) Explicaciones religiosas que se puede referir a "las causas de la homosexualidad están relacionadas con el incumplimiento de la palabra de Dios"; y (d) Explicaciones psicosociales: Por ejemplo "las causas de la homosexualidad están relacionadas con experiencias traumáticas en la primera infancia" y un quinto factor de filtro para inocular error que incluye propuestas como "conflicto con otros países". El análisis factorial reportó una variabilidad explicada en un 59\%, incluyendo las cinco explicaciones. La consistencia interna de los ítems, especialmente las explicaciones éticas $(\alpha=.89)$ y las explicaciones religiosas ( $\alpha=.81$ ) fueron fiables; los valores de Cronbach de las explicaciones psicosociales ( $\alpha=.76)$ y las explicaciones psicológicas ( $\alpha=073$ ) fueron aceptables. Los ítems de las explicaciones biológicas tuvieron una consistencia interna dudosa $(\alpha=.62)$.

Escala de actitudes emocionales. Cada ítem contenía una palabra que representaba una actitud emocional (por ejemplo, afecto, admiración o cordialidad). Los participantes debían indicar con qué frecuencia presentaban cada una de estas actitudes en relación con las personas homosexuales, con una escala de 1 a 5 . La escala de actitud hacia la homosexualidad presentó una estructura unidimensional por ejes principales y el criterio de auto valores mayores a 1 (criterio KaiserGuttman), explicando $41 \%$ de la varianza total. Su consistencia interna fue de \pm .87 y su distribución se ajustó a una curva normal (Moral, 2009)

Escala de percepción personal. Se encargó de examinar las percepciones personales de los participantes a través de seis ítems con atributos personales (por ejemplo "Cumple con los demás, servicial" u "Orientado/a al éxito, al triunfo"). La escala de respuesta contuvo números de 1 (Nada característico) a 5 (Muy característico). El análisis factorial de máxima verosimilitud indicó que se trata de un solo factor que explica el 40 por ciento de la varianza con un alpha de .76.

\section{Procedimiento}

Los cuestionarios fueron aplicados durante los meses de julio y agosto de 2017 en el tiempo curricular de los estudiantes, por lo tanto, se requería la autorización de los docentes encargados de cada asignatura para realizar la ejecución de la investigación. Los participantes diligenciaron el consentimiento informado para continuar con el cuestionario de manera individual, el cual tenía una duración de 15 a 20 minutos.

\section{Análisis de datos}

Para el análisis de la información, se aplicó la prueba t-student que permite comparar grupos y variables independientes. De acuerdo con el presente estudio, fueron: percepción personal, género e ingresos familiares en relación con las variables dependientes asociadas a la homofobia. Asimismo, se utilizó la prueba de ANOVA para grupos independientes, y para este caso se tomaron los grupos de estudiantes por facultades universitarias con homofobia (rechazo a la intimidad y escala de explicaciones de la homosexualidad). Las dos pruebas fueron realizadas a través del programa estadístico SPSS 20.

\section{Consideraciones Éticas}

El estudio tuvo en cuenta y se rigió bajo las consideraciones éticas del país, las cuales son la Resolución 8430 de 1993 del Ministerio de Salud y la Ley 1090 de 2006 (Congreso de la República), que orientan las normas científicas, técnicas y administrativas para la investigación en salud en seres humanos y el ejercicio profesional del psicólogo en Colombia. Por lo tanto los participantes firmaron un consentimiento informado previo donde comunican y aceptan la participacion voluntaria en la investigación. Igualmente, la investigación fue avalada por el Comité de ética de Investigación en la institución participante.

\section{RESULTADOS}

\section{Percepción personal y homofobia}

Aplicando la prueba t-student para grupos y variables independientes, los/as participantes con las medias más altas en la escala de la percepción personal mostraron significativamente ( $a \leq .05)$ menos 
incomodidad al enfrentar situaciones sociales con personas de preferencias homosexuales $(t=2.7$; $g l=222$; $p=.008)$ y asociaron significativamente más emociones positivas hacia la homosexualidad ( $\mathrm{t}=4.70 ; \mathrm{gl}=219$; $p=.000)$.

\section{Género y homofobia}

Los hombres tuvieron un valor de diferencia entre medias mayor en la escala del rechazo a la intimidad $(t=5.6 ; g l=218 ; p=.000)$, comparado con la escala de la expresión emocional ( $\mathrm{t}=2.9 ; \mathrm{gl}=218 ; p=.004)$ y la escala de las causas de la homosexualidad ( $\mathrm{t}=2.8 ; \mathrm{gl}=219 ; p=.007$ ) que las mujeres. Lo anterior traduce que los hombres asociaron más incomodidad y emociones negativas a la homosexualidad; además, reportaron que la causa de la homosexualidad se relaciona con explicaciones externas.

\section{Ingresos familiares y homofobia}

En la escala del rechazo a la intimidad y en la escala de la expresión emocional hacia la homosexualidad se pudo observar que los/as participantes con ingresos familiares de más de 10 SMMLV (la categoría más alta de los ingresos familiares) mostraron los puntajes homofóbicos más altos que los participantes con ingresos familiares más bajos. Sin embargo, esas tendencias no fueron significantes en las pruebas de contraste $(\alpha \leq .05)$.

\section{Facultad universitaria y homofobia}

Aplicando la prueba de ANOVA para grupos independientes, los participantes de las Facultades de Ciencias Económicas y Administrativas y de Ingeniería, mostraron significativamente ( $a \leq .05)$ más incomodidad, especialmente con las siguientes situaciones; "Si su mejor amigo/a le dice que es gay" ( $\mathrm{F}=6.45, p=.000)$ y "Tener un amigo homosexual" ( $\mathrm{F}=4.84, p=.003)$. Una vez se determinó que existen diferencias entre las medias, las pruebas de rango post hoc y las comparaciones múltiples por parejas permitieron determinar qué medias difieren. En la Tabla 1 se presentan las pruebas de rango identificando los dos subconjuntos homogéneos de medias que son significativamente diferentes a un nivel alfa de .05. Se observó que los alumnos de ciencias económicas - y administrativas e ingeniería presentaron las medias más altas de incomodidad "Si su mejor amigo/a le dice que es gay" y "Tener un amigo homosexual"
Tabla 1. Prueba post hoc Scheffé para el contraste de medias de la escala de rechazo a la intimidad y el factor división de estudios del alumno

\begin{tabular}{lccccc}
\hline \multirow{2}{*}{ Facultad } & & \multicolumn{2}{c}{ S1 } & \multicolumn{2}{c}{ S2 } \\
\cline { 3 - 6 } & $n$ & Ml & NMI & Ml & NMI \\
\hline $\begin{array}{l}\text { Ciencias de la } \\
\text { Salud }\end{array}$ & 41 & 1.23 & & 1.10 & \\
$\begin{array}{l}\text { Humanidades y } \\
\text { Ciencias sociales }\end{array}$ & 81 & & & & \\
$\begin{array}{l}\text { Ciencias } \\
\text { Económicas y }\end{array}$ & 89 & 1.27 & & 1.17 & 1.17 \\
$\begin{array}{l}\text { Administrativas } \\
\text { Ingeniería }\end{array}$ & 17 & 1.74 & 1.74 & 1.48 & 1.48 \\
\hline
\end{tabular}

Nota:S1 =Situación 1 "Si su mejor amigo/a le dice que es gay"; S2=Situación

2 "Tener un amigo homosexual"; $\mathrm{MI}=\mathrm{Me}$ incomoda; $\mathrm{NMI}=\mathrm{No}$ me incomoda

Por último, se observó que los estudiantes de las facultades de Ciencias Económicas y Administrativas y de Ingeniería relacionaron la homosexualidad por explicaciones externas, como la falta de fe religiosa $(\mathrm{F}=$ $4.9, p=.003)$, traumas en la primera infancia $(\mathrm{F}=4.94, p$ $=.002)$ y abuso sexual en la infancia $(\mathrm{F}=6.24, p=.000)$ en comparación con los/as participantes que están inscritos en las Facultades de Ciencias de la Salud y de Humanidades y Ciencias Sociales. En la Tabla 2 se presentan los valores de la prueba post hoc únicamente para los subgrupos de comparación de las diferentes divisiones encontrando que para abuso sexual se diferenciaron tres grupos; Ciencias de la Salud, Económico y Administrativas e Ingeniería, mientras que para la creencia de que la homosexualidad está relacionada con traumas en la infancia, la diferencia la tuvieron los alumnos de Ingeniería.

Tabla 2. Prueba post-hoc de Scheffé para dos ítems de la Escala de explicaciones de la homosexualidad

\begin{tabular}{|c|c|c|c|c|c|c|}
\hline \multirow{3}{*}{ Facultad } & \multirow{3}{*}{$n$} & \multicolumn{3}{|c|}{ "Abuso sexual" } & \multicolumn{2}{|c|}{$\begin{array}{l}\text { "Traumas } \\
\text { infancia" }\end{array}$} \\
\hline & & \multicolumn{3}{|c|}{$\begin{array}{c}\text { Subconjunto para } \\
\text { alfa }=.05\end{array}$} & \multicolumn{2}{|c|}{$\begin{array}{l}\text { Subconjuntc } \\
\text { para alfa }=.05\end{array}$} \\
\hline & & 1 & 2 & 3 & 1 & 2 \\
\hline $\begin{array}{l}\text { Ciencias de la } \\
\text { Salud }\end{array}$ & 41 & 1.85 & & & 2.02 & \\
\hline $\begin{array}{l}\text { Humanidades y } \\
\text { Ciencias sociales }\end{array}$ & 81 & 2.16 & 2.16 & & 2.20 & \\
\hline $\begin{array}{l}\text { Ciencias } \\
\text { Económicas y } \\
\text { Administrativas }\end{array}$ & 89 & & 2.61 & 2.61 & 2.60 & 2.60 \\
\hline Ingeniería & 17 & & & 2.94 & & 3.12 \\
\hline
\end{tabular}




\section{DISCUSIÓN}

La presente investigación tuvo como objetivo explorar las percepciones y actitudes sobre la homofobia en estudiantes universitarios. La teoría de la mentalización describe una metacognición que permite la reflexión de construcciones mentales (Wyl, 2014), como el autoconcepto. Los seres humanos que tienen un autoconcepto positivo lo transmiten y lo expresan automáticamente también a las demás personas. El presente estudio evidenció que las personas que reportaron un autoconcepto positivo tuvieron menores actitudes de homofobia. Este proceso lleva una predisposición más baja para desarrollar actitudes homofóbicas, las cuales integran asociaciones y opiniones negativas hacia las personas LGB. En contraste, las personas que tienen un autoconcepto negativo, tienden a tener actitudes negativas hacia otras personas, especialmente hacia personas con una orientación homosexual. Sería interesante investigar si el autoconcepto que está formado en la experiencia influye también la parte subjetiva de la homofobia formando estudios con el IAT. Para responder esa pregunta es esencial saber que las partes objetiva y subjetiva de la homofobia no están correlacionadas (Steffens, 2005).

De acuerdo con la investigación desarrollada por Snowden \& Gray (2013), las mujeres heterosexuales no tienen una atracción sexual específica al género, mientras los hombres heterosexuales tienen una atracción sexual más específica, por lo cual esto puede explicar que las mujeres indican una puntuación más baja en homofobia. También existen teorías descriptivas sobre la diferencia del género con respecto a las actitudes homofóbicas. Por ejemplo, Hamilton (2007) juntó formulaciones teóricas de investigaciones previas que dicen que la homofobia en hombres existe para fortalecer ideales heteronormativos y mantener la dominancia sobre las mujeres (Da SilvaGusmão et al., 2016). Una explicación similar brinda Norton (1997), quien sugiere que los hombres heterosexuales tienen un temor de la feminización de su masculinidad y del derrumbamiento de la distinción tradicional entre los hombres como superiores y las mujeres como inferiores. Lo anterior evidencia la relación entre homofobia y sexismo, en la cual convergen hostilidades como mecanismo social e ideológico en el que conjugan creencias, valores y sentimientos negativos por la orientación sexual mediante un proceso de subordinación heterocentrista que se manifiesta especialmente a través de actitudes negativas, rechazo, intolerancia, violencia, estigma, discriminación entre otras formas que vulneran socialmente.

Las teorías sociales postulan que las dinámicas del grupo de iguales influyen más en los niños con códigos homofóbicos que las niñas en la adolescencia. Una investigación cuantitativa y cualitativa que hizo Saeteros, Sanabria \& Pérez (2014) encontró que los hombres consideran que las personas homosexuales tienen dicha orientación sexual por costumbres, abuso, maltrato en la infancia, en la línea del estudio de Plummer (2000) en el que se asociaba a las personas gais con: ser bebé, ser tímido, ser artístico y lo más significativo, no estar integrado en la cultura de los iguales. Adicionalmente, los estudios reconocen la relación entre procesos de estigmatización, mitos y estereotipos, teniendo presente que son consecuencia de los fenómenos socio-culturales como el patriarcado y el heterocentrismo que permea la trasmisión de modelos diferentes de masculinidad y feminidad en hombres y mujeres, los cuales inician desde edades tempranas en los diferentes espacios de socialización primaria y secundaria que generan como consecuencia la presencia de actitudes de rechazo y discriminación. De igual manera, la investigación de Rodríguez \& Treviño (2016) revela que en el ámbito educativo existen actitudes sexistas y de rechazo hacia la diversidad sexual, identificándose mayores niveles de sexismo que de rechazo hacia las personas LGTBI.

En el presente estudio, respecto a las características sociodemográfica de ingresos económicos, los estudiantes de más altos ingresos presentaron medias más altas en homofobia, aunque no estadísticamente significativas comparadas con los de bajos ingresos. En esta misma línea, en un estudio realizado en Paraguay, no se encontraron diferencias en los puntajes de homofobia entre los estudiantes de la universidad pública y la universidad privada y tampoco entre las áreas rurales y las áreas urbanas, los cuales implican diferencias de ingresos (Coppari et al., 2014).

Las tendencias de los participantes de este estudio, con ingresos familiares altos hacia la homofobia puede ser explicada con la hipótesis de que las personas con ingresos altos dan más atención a cumplir normas sociales, por ejemplo, ser heterosexual; además, relacionan la homosexualidad con estratos socioeconómicos más bajos. En cuanto al género, no se 
evidenció una diferencia. Por su parte, la oscilación de la edad fue pequeña para mostrar diferencias; se agruparon entre los 18 a 20 años y los 21 a 24 años, es por ello que se recomienda para futuras investigaciones abordar la homofobia en personas de diferentes edades para generar una comparación por grupos etarios. Asimismo, se encuentra pertinente analizar la religión y la raza con la homofobia para futuros estudios, pues de acuerdo con la investigación desarrollada por Roggemans, Spruyt, Van Droogenbroek \& Keppens (2015) cuando se practica una religión y se es parte de una población racial minoritaria, hay mayor tendencia hacia la actitudes y percepciones homofóbicas.

En cuanto a la existencia de diferencias en la homofobia acorde con la carrera profesional, se evidenció una diferencia signficativa entre ciertos programas académicos como Ingeniería respecto a otros como los que se encuentran dentro de las Humanidades y Ciencias Sociales. Lo anterior concuerda con un estudio realizado en Brasil en el año 2002 (usando las mismas escalas de medidas), donde también se evidenciaron diferencias significativas en la puntuación de la homofobia dependiendo de la carrera de los estudiantes universitarios. Coincidiendo como el presente estudio, los estudiantes de Ingeniería tuvieron un nivel de homofobia más alto mientras que los estudiantes de Ciencias Sociales mostraron el nivel más bajo. A diferencia del método de análisis de esta investigación, Lacerda et al. (2002) usaron un análisis de cluster para formar tres grupos de estudiantes con diferentes niveles de homofobia y mostraron también que el grupo "flagrante en homofobia" tiende a reconocer la homofobia con explicaciones internas como biológicas y religiosas. Al contrario, el grupo de "bajo prejuicio", abordan la homofobia con explicaciones externas como el entorno social.

Por último, esta investigación aporta elementos para futuras investigaciones que incluyan un enfoque de intervención y prevención, desde los ámbitos de salud y educación, para mitigar las actitudes, percepciones e interacciones entre personas heterosexuales y de orientaciones sexuales no hegemónicas como gais, lesbianas y bisexuales, con el propósito de promover la igualdad y equidad de las personas LGB. Uno de los escenarios que requiere ser priorizado son los contextos educativos y familiares, teniendo presente que son los principales agentes de socialización que permean actitudes, creencias, valores asociados a la diversidad sexual y de géneros. Lo anterior implica el diseño de estrategias de intervención que favorezcan espacios de respeto, inclusión, aceptación e igualdad en las relaciones humanas con énfasis en el reconocimiento de la construcción social del género. En el contexto universitario, se recomienda la inclusión de asignaturas que aborden la diversidad sexual y de géneros como aspecto clave en la formación integral académica y humana, que deconstruya las representaciones e imaginarios sociales que afectan actualmente a la sociedad ante la presencia de actitudes homofóbicas, sexistas y de discriminación por la identidad y orientación sexual.

\section{Conflicto de interés}

Los autores no tienen conflictos de intereses que declarar.

\section{REFERENCIAS}

Aggleton, P., \& Parker, R. (2002). Estigma y discriminación relacionados con el VIHISIDA. Marco conceptual y base para la acción. Ginebra: ONUDIDA.

Andreou, E., Didaskalou, E., \& Vlachou A. (2008) Outcomes of a curriculumbased anti-bullying intervention program on students' attitudes and behavior. Emotional Behavioural Difficulties., 13(4), 235-49.

António, R., \& Moleiro, C. (2015). Social and parental support as moderators of the efects of homophobic bullying on psychologial distress in youth. Psychology in the Schools, 52(8), 729-742. doi:10.1002/pits.21856

Badenes-Ribera, L., Sánchez-Meca, J., \& Longobardi, C. (2017). The relationship between internalized homophobia and intimate partner violence in same-sex relationships: a meta-analysis. Trauma, Violence, \& Abuse, XX(X), 1-13. doi:10.1177/1524838017708781

Boysen, G. A., Vogel, D. L., \& Madon, S. (2006). A public versus private administration of the implicit association test. European Journal of Social Psychology, 36(6), 845-856. doi:10.1002/ejsp.318

Coppari, N., Arcondo, G., Bagnoli, L., Chaves, M., Corvalán, M., Enciso, A., ... Rodríguez, X. (2014). Prejuicio y distancia social hacia la homosexualidad en universitarios de psicología de Paraguay [Prejudice and social distance to homosexuality in Psychology students of Paraguay]. Salud \& Sociedad, 5(3), 240-252. doi:10.22199/S07187475.2014.0003.00001

Cornejo, J. (2007). La homosexualidad como construcción ideológica. Límite: Revista de Filosofía y Psicología, 16, 83-108. 
Costa, P. A., \& Davies, M. (2012). Portuguese adolescents' attitudes toward sexual minorities: Transphobia, homophobia, and gender role beliefs. Journal of homosexuality, 59(10), 1424-1442. doi:10.1080/00918369.2012.724944

Da Silva-Gusmão, E.E., da Silva-Nascimento, B., Veloso, V., Ferreira, L. G., Rodrigues, K. M., de Moura, H. M., \& Pereira, R. (2016). Valores Humanos e Atitudes Homofóbicas Flagrante e Sutil. Psico-USF, 21(2), 367-380. doi:10.1590/1413-82712016210213

Dempsey, D., Hillier, L., \& Harrison, L. (2002). Gendered (s)explorations among same-sex attracted young people in Australia. Journal of Adolescence, 24(1), 67-81

Dijker, A. J. (1987). Emotional reactions to ethnic minorities. European Journal of Social Psychology, 17(3), 305-325. doi:10.1002/ejsp.2420170306

Evans, C.B.R., Smokowski, P.R., \& Cotter, K,L. (2014). Cumulative bullying victimization: an investigation of the dose-response relationship between victimization and the associated mental health outcomes, social supports, and school experiences of rural adolescents. Child Youth Services Review, 44, 256-64.

Mastropasqua, K. (2015). "Discrimination and Violence against Individuals Based on their Sexual Orientation and Gender Identity," United Nations Human Rights Council Report. Recuperado de https://www.ohchr.org/Documents/Issues/Discrimination/LGBT/A HRC_29_23_One_pager_en.pdf

Moral de la Rubia, José. (2009). Conducta homosexual en estudiantes universitarios y aspectos diferenciales de género. La ventana. Revista de estudios de género, 3(29), 206-235.

Fingerhut, A. W., Peplau, L. A., \& Ghavami, N. (2005). A dual-identity framework for understanding lesbian experience. Psychology of Women Quarterly, 29, 129-139. doi:10.1111/j.1471$\underline{6402.2005 .00175 . x}$

Flowers, P., \& Buston, K. (2001) I was terrified of being different": exploring gay men's accounts of growing-up in a heterosexist society. Journal of Adolescence, 24(1), 51-65. doi:10.1006/jado.2000.0362

Formby, E. (2011). Sex and relationships education, sexual health, and lesbian, gay and bisexual sexual cultures: views from young people. Sex education, 11(3), 255-266. doi:10.1080/14681811.2011.590078

Hamilton, L. (2007). Trading on heterosexuality: College women's gender strategies and homophobia. Gender \& Society, 21(2), 145-172. doi:10.1177/0891243206297604

Hatzenbuehler, M. L., McLaughlin, K. A., Keyes, K. M., \& Hasin, D. S. (2010). The impact of institutional discrimination on psychiatric disorder in lesbian, gay, and bisexual populations: A prospective study. American Journal of Public Health, 100, 452-459. doi:10.2105/AJPH.2009.168815

Hernández-Sampieri, S., Fernández, C., \& Baptista, P. (2010). Metodología de la investigación. México D.F, Mexico: McGraw-Hill.

Herrick, A. L., Stall, R., Chmiel, J. S., Guadamuz, T. E., Penniman, T., Shoptaw, S., ... Plankey, M. W. (2013). It gets better: resolution of internalized homophobia over time and associations with positive health outcomes among MSM. AIDS and Behavior, 174), 14231430. doi:10.1007/s10461-012-0392-x

Jeffries, W. L., Marks, G., Lauby, J., Murrill, C. S., \& Millett, G. A. (2013). Homophobia is associated with sexual behavior that increases risk of acquiring and transmitting HIV infection among black men who have sex with men. AIDS and Behavior, 17(4), 1442-1453. doi:10.1007/s10461-012-0189-y

Junqueira, R. D. (2012). Homofobia: limites e possibilidades de um conceito em meio a disputas [Homophobia: limits and possibilities of a concept in the midst of disputes]. Bagoas-Estudos gays. gêneros e sexualidades, 1(01), 145-166.

Kelly, J., David, C., \& Schlesinger, C. (2015). Substance use by same sex attracted Young people: prevalence, percepctions and homophobia. Drug and Alcohol Review, 34, 358-365. doi:10.1111/dar.12158

Lacerda, M., Pereira, C., \& Camino, L. (2002). Um estudo sobre as formas de preconceito contra homossexuais na perspectiva das representações sociais [A Study of Prejudice Forms against Homosexuals Anchored on Social Representations]. Psicologia: reflexão e crítica, 15(1). doi:10.1590/S0102-79722002000100018.

Mahaffey, A. L., Bryan, A. D., Ito, T. A., \& Hutchison, K. E. (2011). In search of the defensive function of sexual prejudice: Exploring antigay bias through shorter and longer lead startle eye blink. Journal of Applied Social Psychology, 41(1), 27-44. doi:10.1111/j.15591816.2010.00700.x

Meyer, I. H., \& Dean, L. (1998). Internalized homophobia, intimacy, and sexual behavior among gay and bisexual men. En G. M. Herek (Ed), Stigma and sexual orientation: Understanding prejudice against lesbians, gay men, and bisexuals (pp. 160-186). Thousand Oaks, CA, US: Sage Publications, Inc. doi:10.4135/9781452243818.n8

Moral, J., \& Valle, A. (2014). Predictive models of attitude toward homosexuality in heterosexual men. Universitas Psychologica, 13(4), 1395-1407. doi:10.11144/Javeriana.UPSY13-4.pmah

Nadal, K. L., \& Griffin, K. E. (2011). Microaggressions: A root of bullying, violence, and victimization toward lesbian, gay, bisexual, and transgender youths. In M. A. Paludi (Ed.), The psychology of teen violence and victimization. (pp. 3-22). Santa Barbara, CA: Praeger.

Nagoshi, J. L., Adams, K. A., Terrell, H. K., Hill, E. D., Brzuzy, S., \& Nagoshi, C. T. (2008). Gender differences in correlates of homophobia and transphobia. Sex Roles, 59, 521-531. doi:10.1007/s11199-008-9458-

Navarro, M. C., \& Mebarak, M. (2014). Formación de prejuicios sociales: una revisión desde el inconsciente cognitivo y psicoanalítico [Formation of Social Prejudice, A Review from the Cognitive and Psychoanalytic Unconscious]. Cuadernos de Neuropsicología, 8(19), 88-106.

Norton, J. (1997). "Brain says you're a girl, but I think you're a sissy boy": Cultural origins of transphobia. International Journal of Sexuality and Gender Studies, 2(2), 139-164. doi:10.1023/A:1026320611878

O'Malley Olsen, E., Kann, L., Vivolo-Kantor, A., Kinchen, S., \& McManus, T. (2014). School violence and bullying among sexual minority high 
school students, 2009-2011. Journal of Adolescent Health. doi:10.1016/j.jadohealth.2014.03.002

Pettigrew, T. F., \& Meertens, R. W. (1995). Subtle and blatant prejudice in Western Europe. European journal of social psychology, 25(1), 5775. doi:10.1002/ejsp.2420250106

Plummer, D. C. (2001). The quest for modern manhood: Masculine stereotypes, peer culture and the social significance of homophobia. Journal of adolescence, 24(1), 15-23. doi:10.1006/jado.2000.0370

Prado, M., Aurélio, M., Mountian, I., Viana Machado, F., \& Cardoso dos Santos, L. (2010). Los movimientos LGTB y la lucha por la democratización de las jerarquias sexuales en Brasil [LGBT movements and struggles for the democratization of sexual hierarchies in Brazil]. Revista Digital Universitaria, 11(7), 1-15.

Rodríguez Otero, L. M. (2018). Bullying homofóbico en México a nivel de secundaria: el contexto de Nuevo León. Revista de Psicología (PUCP), 36(2), 631-659.

Rodríguez-Otero, L. M., \& Treviño-Martínez, L. (2016). Sexismo y actitudes hacia la homosexualidad, la bisexuaidad y la transexualidad en estudiantes de Trabajo Social mexicanos. Trabajo Social GlobalGlobal Social Work, 6(11), 3-30.

Roggemans, L., Spruyt, B., Van Droogenbroeck, F., \& Keppens, G. (2015). Religion and negative attitudes towards homosexuals: an analysis of urban young people and their attitudes towards homosexuality. Young, 23(3), 254-276. doi:10.1177/1103308815586903

Rohner, J. C., \& Björklund, F. (2006). Do self-presentation concerns moderate the relationship between implicit and explicit homonegativity measures? Scandinavian Journal of Psychology, 475), 379-385. doi:10.1111/j.1467-9450.2006.00522.x

Saeteros, R. C., Sanabria, G., \& Pérez, J. (2014). Imaginario sobre la homosexualidad en estudiantes de politécnicos ecuatorianos [Imaginary on homosexuality ecuatorian polytechnic students]. Revista Cubana de Salud Pública, 40(4), 299-313.

Shilo, G., \& Mor, Zohar. (2014). The impact of minority stressors on the mental and physical health of lesbian, gay, and bisexual youths and young adults. Health \& Social Work, 39(3), 161-171.

Silva, P., Jaeger, A. A. \& Valdivia-Moral. (2018). Percepción de los estudiantes sobre comportamientos homofóbicos y heterosexistas en educación física. Revista de Psicología del Deporte, 27(2). 39-46.

Snowden, R. J., \& Gray, N. S. (2013). Implicit sexual associations in heterosexual and homosexual women and men. Archives of Sexual Behavior, 42(3), 475-485. doi:10.1007/s10508-012-9920-z

Steffens, M. C. (2005). Implicit and explicit attitudes towards lesbians and gay men. Journal of Homosexuality, 49(2), 39-66. doi:10.1300/J082v49n02_03

Tsang, J. A. \& Rowatt, W. C. (2007). The relationship between religious orientation, right-wing authoritarianism, and implicit sexual prejudice. The international journal for the psychology of religion, 172), 99-120. doi:10.1080/10508610701244122
Umana-Taylor, A. J., \& Updegraff, K,A. (2007) Latino adolescents' mental health: exploring the interrelations among discrimination, ethnic identity, cultural orientation, self-esteem, and depressive symptoms. Journal of Adolescence, 30, 549-67

Varjas, K., Dew, B., Marshal, M. P., Graybill, E., Singh, A., Meyers, J., \& Birckbichler, L. (2008). Bullying in schools towards sexual minority youth. Journal of School Violence, $7(2)$, 59-86. doi:10.1300/J202v07n02 05

Wyl, A. (2014). Mentalisierung und Theory of Mind. Praxis der Kinderpsychologie und Kinderpsychiatrie, 63(9), 730-737. doi:10.13109/prkk.2014.63.9.730 